\title{
Dual-magnetron open field sputtering system for sideways deposition of thin films
}

\author{
Asim Aijaz, Daniel Lundin, Petter Larsson and Ulf Helmersson
}

\section{Linköping University Post Print}

N.B.: When citing this work, cite the original article.

Original Publication:

Asim Aijaz, Daniel Lundin, Petter Larsson and Ulf Helmersson, Dual-magnetron open field sputtering system for sideways deposition of thin films, 2010, SURFACE and COATINGS TECHNOLOGY, (204), 14, 2165-2169.

http://dx.doi.org/10.1016/j.surfcoat.2009.11.044

Copyright: Elsevier

http://www.elsevier.com/

Postprint available at: Linköping University Electronic Press

http://urn.kb.se/resolve?urn=urn:nbn:se:liu:diva-54766 


\section{Accepted Manuscript}

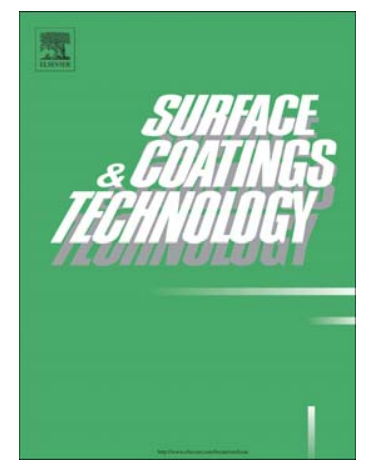

Dual-magnetron open field sputtering system for sideways deposition of thin films

Asim Aijaz, Daniel Lundin, Petter Larsson, Ulf Helmersson

PII:

S0257-8972(09)00997-9

DOI: $\quad$ doi: $10.1016 / j$.surfcoat.2009.11.044

Reference: $\quad$ SCT 15435

To appear in: $\quad$ Surface \& Coatings Technology

Received date: 6 October 2009

Accepted date: 27 November 2009

Please cite this article as: Asim Aijaz, Daniel Lundin, Petter Larsson, Ulf Helmersson, Dual-magnetron open field sputtering system for sideways deposition of thin films, Surface \& Coatings Technology (2009), doi: 10.1016/j.surfcoat.2009.11.044

This is a PDF file of an unedited manuscript that has been accepted for publication. As a service to our customers we are providing this early version of the manuscript. The manuscript will undergo copyediting, typesetting, and review of the resulting proof before it is published in its final form. Please note that during the production process errors may be discovered which could affect the content, and all legal disclaimers that apply to the journal pertain. 


\title{
Dual-magnetron open field sputtering system for sideways deposition of thin films
}

\author{
Asim Aijaz*, Daniel Lundin, Petter Larsson, and Ulf Helmersson \\ Plasma \& Coatings Physics Division, IFM-Materials Physics, Linköping University, \\ SE-581 83 Linköping, Sweden
}

* Corresponding Author. E-mail: asim@ifm.liu.se

Complete Address: Asim Aijaz

Department of Physics, Chemistry \& Biology,

Linköping University,

SE-581 83, Linköping,

Sweden.

Office Phone:

0046-13-286616

Fax:

0046-13-137568

\begin{abstract}
A dual-magnetron system for deposition inside tubular substrates has been developed. The two magnetrons are facing each other and have opposing magnetic fields forcing electrons and thereby also ionized material to be transported radially towards the substrate. The depositions were made employing direct current magnetron sputtering (DCMS) and high power impulse magnetron sputtering (HiPIMS). To optimize the deposition rate, the system was characterized at different separation distances between the magnetrons under the same sputtering conditions. The deposition rate is found to increase with increasing separation distance independent of discharge technique. The emission spectrum from the HiPIMS plasma shows a highly ionized fraction of the sputtered material. The electron densities of the order of $10^{16} \mathrm{~m}^{-3}$ and $10^{18} \mathrm{~m}^{-3}$ have been determined in the DCMS and the HiPIMS plasma discharges respectively. The results demonstrate a successful implementation of the concept of sideways deposition of thin films providing a solution for coating complex shaped surfaces.
\end{abstract}

Keywords: Dual-magnetron; Open field configuration; Sideways deposition; HiPIMS; HPPMS; DCMS 


\section{Introduction}

The rapid development of the thin film industry has been leading material scientists and material processing engineers to focus not only on the study of new and multi-functional materials but also motivating them to improve existing systems and to develop new deposition techniques. The concept of an unbalanced magnetron provided by Window and Savvides [1] can be regarded as the first step towards an improvement of a conventional single-cathode balanced magnetron sputtering system. An unbalanced magnetic field configuration showed promising improvements $[1,2,3]$ and led Sproul et al. to construct a dual-cathode, high sputtering rate, unbalanced magnetron sputtering system $[4,5]$. They made studies on different magnetic field configurations referred to as closed field and open field configurations [4]. In a closed field dual-cathode unbalanced magnetron sputtering system two opposing magnetrons are configured with magnets of opposite polarity, and thereby forming closed traps for electrons in the plasma. On the other hand, when two opposing magnetrons are configured with magnets of identical polarity it is called an open field configuration [6]. Closed field multi-cathode sputtering systems have attracted much attention compared to open field systems, since they offer a dramatic increase of ion-bombardment on the substrates and the growing film. This is due to the extension of the plasma towards the substrate in this type of configuration, which increases the ion-bombardment of the growing film. The details of these systems can be found elsewhere [6].

One of the shortcomings of an open field configuration is that the magnetic field lines are directed to the sides, often towards the chamber walls. Electrons following these lines are lost and the plasma is extended towards the chamber walls instead of the position parallel to the cathode (facing the cathode), where a substrate usually is placed. This suggests that a substrate placed perpendicular to the surface of the magnetrons (not facing the cathode) in an open field configuration will be subjected to significant ion-bombardment since the plasma is extended in that direction. Still, the disadvantage with this approach is the low deposition rate 
for a substrate oriented perpendicular to the cathode surface and placed at the rim, or even further away from the magnetron since most of the deposition material is ejected close to the normal direction from the sputtering target, following a cosine distribution. In the case of ionized physical vapour deposition (IPVD) techniques, such as high power impulse magnetron sputtering (HiPIMS), which can provide a plasma discharge with a high ionized fraction of sputtered material [7] the situation changes. Although the magnetic field of the magnetrons is often too weak for the ions to become magnetized, the flux of electrons to the side will generate the driving force for ions to move with the electrons to maintain the quasineutrality nature of the plasma. The choice of HiPIMS may prove to be of particular interest for implementing the concept of sideways deposition of thin films, since recently an enhanced side-transport mechanism of sputtered and ionized material has been discovered by Lundin $e t$ al. in this regime [8]. In this study it is shown that a large flux of sputtered material is transported sideways (up to $80 \%$ of the deposition rate for a substrate placed at a conventional position, i.e. facing the cathode, in the case of titanium). Furthermore, mass spectrometry measurements of the flux showed a high-energy tail of the ion energy distribution, which is believed to be beneficial for thin film growth, since it promotes increased ad-atom mobility.

In the present study the previously reported side-transport in HiPIMS is utilized for film growth. A dual-magnetron open field sputtering system has been developed, employing two identical target cathodes, with the aim to grow high quality thin films at a reasonable deposition rate under different discharge conditions. Both target cathodes were driven synchronously at the same voltage. The separation distance between the magnetrons was varied to optimize the deposition rate. The microstructure of the resulting films was investigated by scanning electron microscope (SEM). Optical emission spectroscopy (OES) was employed to investigate the ionized fraction of the sputtered material. A Langmuir probe was used to determine the electron density by determining the electron energy distribution 
function (EEDF) [9]. For the sake of comparison, all of the measurements were also repeated with conventional direct current magnetron sputtering (DCMS).

\section{Experimental details}

The dual-magnetron open field sputtering system used in this work was constructed by mounting two identical planar circular unbalanced magnetrons, each with a diameter of $7 \mathrm{~cm}$, facing each other co-axially in a cylindrical vacuum chamber (height $30 \mathrm{~cm}$, diameter $42 \mathrm{~cm}$ ). One of the magnetrons was mounted in the lid and the other in the base of the chamber as shown in figure 1 . Both of the magnetrons were equipped with identical $5 \mathrm{~cm}$ Ti targets ( $99.9 \%$ purity). The separation distance between the magnetrons was set to 3,5 , and $8 \mathrm{~cm}$. A static and grounded substrate holder with three Si substrates mounted vertically was placed 2 $\mathrm{cm}$ from the edge of the magnetrons. The center of the separation distance between the magnetrons was chosen as a reference point for all measurements, as indicated by the origin of the coordinate axes in figure 1. A simple model of the magnetic field configuration of the dual-magnetron system is presented in figure 2 . The model was constructed using a software package [10]. It assumes the magnetic field strength of both the outer and inner magnets to be

$1 \mathrm{~T}$. It should be noted that the model only provides an estimation of the actual magnetic field configuration but will nonetheless serve its purpose as guidance in the discussions.

Prior to the measurements, the chamber was evacuated to a base pressure of the order of $10^{-6}$ Torr, after which Ar of purity $99.9997 \%$ was introduced through a leak valve to serve the purpose of a sputtering gas at a pressure of 5, 10, and 20 mTorr. For the DCMS measurements both magnetrons were synchronously driven by an MDX $1 \mathrm{~K}$ direct current (DC) power supply (Advanced Energy) operating in constant power mode, whereas for the HiPIMS measurements a pulsing unit (SPIK 1000A, Melec) fed by a DC power supply was used. The pulsing unit produces approximately square voltage pulses of controlled length and repetition frequency. A repetition frequency of $100 \mathrm{~Hz}$ and a pulse width of $100 \mu \mathrm{s}$ corresponding to voltage pulses of $1 \%$ duty cycle were used for all HiPIMS experiments. An 
average power of $250 \mathrm{~W}$ in the case of HiPIMS and a constant power of $250 \mathrm{~W}$ in the case of DCMS were applied to the two magnetrons.

A SEM (LEO 1550 Gemini SEM) was employed for the microstructure analysis of the films and for determining the deposition rates by measuring the thickness. For investigating the ionized fraction of the sputtered material, time-averaged optical emission spectroscopic (OES) measurements were made. In this case, the emission from the plasma was recorded through a side window of the chamber by a Mechelle Sensicam 900 spectrometer connected to a collimator via an optical fiber. The collimator was focused at the reference position. The spectrometer was equipped with a charge coupled device camera capable of measuring a wavelength spectrum in the range $300-1100 \mathrm{~nm}$.

The determination of the plasma parameters such as the plasma density and the plasma potential was made by using a cylindrical shaped Langmuir probe made of a thin tungsten wire. The probe was encapsulated in a ceramic tube with a protruding probe tip of $5 \mathrm{~mm}$ length and a diameter of $125 \mu \mathrm{m}$. The probe was placed parallel to the target surfaces such that the probe tip was positioned at the common axis of the magnetrons. The probe tip had a fixed position which means that the tip of the probe was slightly offset from the reference position, when the separation distance between the magnetrons was varied. The other end of the probe was connected to the external circuitry for applying the bias probe voltage and measuring the resulting current drawn by the probe from the plasma body. The probe bias voltage $V_{B}$ was in all measurements varied in steps of $0.1 \mathrm{~V}$ ranging from $-40 \mathrm{~V}$ to $+15 \mathrm{~V}$.

Due to the pulsed nature of the HiPIMS discharge there is a temporal variation associated with the recorded probe voltage-current characteristics, which should be taken into account. Therefore a time resolved current was recorded using the same trigger signal as used when initiating the HiPIMS voltage pulse. A total of 500 time steps were chosen with an equal interval width of $320 \mathrm{~ns}$. In this way the probe characteristics could be monitored for a total length of $160 \mu$ s after initiating the discharge pulse. For each time value, the I-V curve 
was reconstructed and analyzed. The measured data were in some cases noisy, due to the fact that the plasma itself is inherently noisy. Therefore smoothing and filtering of the data were necessary. Furthermore, even a small low-frequency noise, originating from the discharge itself or from the power supply, reduces the signal-to-noise ratio of the measurements and the dynamic range of the measured electron energy distribution function (EEDF), i.e. the ability to analyze the high energy tail of the EEDF is reduced. Employing averaging techniques over whole data sets helps in overcoming this problem to some extent [11]. In this work an average of 30 samples was used. In order to determine the EEDF needed to calculate the electron density, the second derivative of the I-V curve was calculated by differentiation of the measured data after it had been digitally smoothened using a Blackman window filtration. The details of this method can be found elsewhere [12]. The second derivative of the I-V characteristic curve was used to determine the EEDF numerically from the Druyvesteyn formula [9]:

$$
g_{e}(V)=\frac{2 m}{e^{2} A_{p r}}\left(\frac{2 e V}{m}\right)^{\frac{1}{2}} \frac{d^{2} I_{e}}{d V^{2}} .
$$

$A_{p r}$ is the probe area, $m$ and $e$ are the electron mass and charge, $I_{e}$ is the electron current, and $V=V_{p l}-V_{B}$ is the probe potential with respect to the plasma potential. Once the EEDF is know, the electron density is determined by:

$$
n_{e}=\int_{0}^{\infty} g_{e}(E) d E
$$

where $E$ is the electron energy.

\section{Results}

\subsection{Microstructural analysis \& deposition rates}

SEM micrographs of the DCMS and HiPIMS films deposited at $3 \mathrm{~cm}$ separation distance between the magnetrons are shown in figure 3. These SEM images were taken at the 
bottommost substrate ( 3 substrates were placed on the sample holder mounted vertically as displayed in figure 1). The microstructural analysis of the grown films reveals that both the DCMS and the HiPIMS films exhibit a columnar structure. However, the HiPIMS film appears denser. The columns of the grains in the HiPIMS film have almost no inclination showing that the deposition flux is mainly arriving at normal incidence angle to the substrate surface. The columns of the grains in the DCMS film have an inclination showing that the growth orientation is not perpendicular to the substrate. It should be noted that no substrate bias was used for the deposition of these films, however, the substrate holder was grounded.

The results from the investigation of the deposition rates for various distances between the magnetrons are shown in figure 4 for the DCMS and the HiPIMS depositions. The deposition rate curves in the case of DCMS are flatter as compared to the deposition rate curves of the HiPIMS, where they are more peaked at approximately the center point between the magnetrons. Considering the peak value of each curve, it is found that the deposition rates with DCMS are higher by a factor of approximately 5.4, 7.3, and 8.2 compared to the HiPIMS rates for the distances of 3,5 , and $8 \mathrm{~cm}$ respectively. It is also observed that the deposition rate is increasing when increasing the distance between the magnetrons in both cases.

\subsection{Plasma analysis}

Figure 5 shows optical emission spectra from the DCMS and HiPIMS plasma discharges. A quantitative comparison has so far not been made, but there is a clear trend that the metal emission (below $600 \mathrm{~nm}$ ) dominates over the Ar emission in the HiPIMS spectrum, while the opposite is true for the DCMS case. Furthermore, the metal and Ar emission in the HiPIMS spectrum is dominated by ions in contrast to the case of DCMS, where both regions of the spectrum are dominated by neutrals.

Figure 6 shows the electron density, plotted for nominal Ar gas pressures and for different magnetron separation distance. The HiPIMS discharge shows approximately two orders of magnitude higher electron density for all positions, while a weak trend of increasing 
electron density for higher gas pressures can be observed for both techniques except at $5 \mathrm{~cm}$ separation for HiPIMS. Interestingly, variation of the separation distance has different effects on the electron density for the two different techniques: the highest value for DCMS is observed at $5 \mathrm{~cm}$, a position where the HiPIMS discharge shows the lowest measured value. The highest electron density attained for HiPIMS in this investigation is recorded at a separation distance of $8 \mathrm{~cm}$. Furthermore, the plasma potential, not shown here, was simultaneously measured to be between 1 and $5 \mathrm{~V}$ depending on the different sputtering conditions.

\section{Discussion}

A successful growth of thin films based on radial flux of sputtered material using the techniques of DCMS and HiPIMS is demonstrated by the results presented above. First we discuss the results from the OES measurements. The optical emission from the HiPIMS and DCMS plasma discharges shown in figure 5 show two very different spectra, where the HiPIMS spectrum is dominated by $\mathrm{Ti}$ and $\mathrm{Ti}^{+}$and the DCMS spectrum is dominated by $\mathrm{Ar}$ and $\mathrm{Ar}^{+}$. The dominating metal ion emission in the HiPIMS OES spectrum is due to a higher plasma density in the HiPIMS discharge as compared to the DCMS discharge (regarding the plasma density see figure 6). A high plasma density means a higher probability for ionizing

collisions and excitation of the sputtered material, keeping in mind that ionization and excitation in a high density plasma occurs mainly due to electron momentum transfer [7]. A low plasma density in the DCMS discharge causes the emission from the sputtering gas to dominate over the metal emission. The results from the OES measurements are in a close agreement with the results reported by Bohlmark et al. for Ti [13]. It can therefore be concluded that the present setup using two magnetrons does not diminish the benefits of HiPIMS plasmas regarding high plasma density and a high degree of ionization of the deposition flux. 
We observed some differences between the microstructure of the DCMS and the HiPIMS films, which will be discussed here. The inclined columns of the off-center DCMS film indicate that the sputtered atoms are arriving with an inclination and subsequently depositing onto the substrate following the same direction. This is generally expected for the DCMS films (see for example reference 14). The reason for less or no inclination of the columns in the HiPIMS film can be understood by the effect that the sputtered material in the case of HiPIMS is substantially ionized providing large amounts of positive metal ions. These ions will be accelerated from the plasma potential to the grounded substrate resulting in ions arriving at the substrate surface at an almost perpendicular angle of incidence. Alami et al. [14] reported similar differences between DCMS and HiPIMS regarding columnar tilt, when they deposited Ta films on biased Si substrates placed along a wall of a $2 \mathrm{~cm}$ deep and $1 \mathrm{~cm}$ wide trench. They showed that the films grown using HiPIMS have a smooth surface and a dense crystalline structure with the grains oriented perpendicular to the substrate surface. The DCMS films exhibited a rough surface, pores between the grains and an inclined columnar growth. In the present case no substrate bias was used, but apparently the potential difference between the plasma and the substrate (ground) is enough to achieve a similar result.

We observed a lower deposition rate for HiPIMS in our experiments compared to DCMS for the same sputtering conditions, as seen from figure 4. One possible explanation for the lower deposition rate in the case of HiPIMS is the back-attraction of metal ions to the target as suggested by Christie [15]. There are also a number of other mechanisms that might play a role in the reduction of the deposition rate such as the non-linear increase of the self sputter yield with increasing applied voltage [16], a change in plasma conductivity [17], the effect of the magnetic field arrangement $[18,19]$ etc. It is not the intention in this work to investigate the importance of these mechanisms. Still, it can be concluded that the spatial distribution of the deposition flux is not necessarily the same for DCMS and HiPIMS. The 
reason for low deposition rates in HiPIMS discharges is likely a combination of the above mentioned factors and possibly others.

Another important aspect is the film thickness distribution for the different samples. There is a stronger spatial variation of the deposition material in the case of HiPIMS, as previously stated regarding the results seen in figure 4, where the HiPIMS deposition rate and thereby the thickness of the deposited film varied more depending on substrate. This result stems most likely from the fact that the deposition flux in a HiPIMS discharge mostly consists of ions, which are affected by the magnetic field from the two magnetrons. In the present configuration the magnetic field will be greatly enhanced in the center position from the edge of the magnetrons and radially towards the chamber walls as displayed in figure 2. This means that the electrons will bunch together at these positions and the ions will follow in order to preserve the quasi-neutrality condition, resulting in more peaked deposition rate curves. The reason for a variation in the deposition rate by changing the separation distance between the magnetrons is not obvious at this point. However, it may be speculated that this variation is associated with the change in the magnetic field configuration when the two magnetrons are brought closer to each other or separated from each other. Another effect which may have an influence on the deposition rate variations in the case of DCMS and HiPIMS, when changing the separation distance is the shadowing of the two magnetrons. From figure 4 it is seen that the deposition rate is less reduced in the HiPIMS case compared to DCMS when decreasing the separation distance. It is likely that the shadowing effects are more pronounced for neutral flux causing a dramatic decrease in the deposition rate. In the HiPIMS case, in addition to following the field lines and reaching the centre point, the ionized flux can also be transported across the magnetic field lines (see for example reference 8 regarding cross-field transport of charged particles) and then guided radially outwards by the magnetic field lines, a process which is less affected by shadowing. 
Last, regarding the electron density shown in figure 6 for the DCMS and HiPIMS plasma discharges, a weak trend of an increase in the electron density with an increase in the gas pressure is observed. This can be understood since an increase in the gas pressure causes a reduction of the mean free path of electrons. This leads to more frequent ionizing collisions of electrons with gas atoms and less diffusion of electrons to the grounded chamber walls. Overall, this results in an increase in the electron density. When it comes to the change in the electron density with respect to separation distance we cannot explain the two different trends for DCMS and HiPIMS. It is likely that a part of the observed variations is caused by the uncertain probe position relative to the reference position. Furthermore, these values may be influenced by the resulting magnetic field when the two magnetrons are brought close together.

\section{Conclusions}

A new technique for sputter depositing thin films has successfully been developed with two magnetrons forming an open field configuration. The sideways depositions of thin films were made using HiPIMS and DCMS. The microstructure analysis reveals that the films are of good quality and have nowhere peeled off the substrate for any of the grown samples. Furthermore, the HiPIMS films are denser as compared to the DCMS films. This is in agreement with the work reported by other authors. The deposition rate of the film growth increased with increased separation distance between the magnetrons for both of the techniques. It was found from the emission spectroscopic studies that the HiPIMS plasma discharge contains a highly ionized fraction of the sputtered material compared to the DCMS plasma. This indicates a higher degree of ionization in the case of the HiPIMS discharge. The plasma densities obtained by Langmuir probe measurements are comparable to typical plasma densities associated with HiPIMS and DCMS discharges. 


\section{Acknowledgements}

The authors are grateful to the Swedish Research Council (VR) and the Swedish Science Foundation (SSF) for providing the financial support for this project. 


\section{References}

[1] B. Window, N. Savvides, J. Vac. Sci. Technol. A 4 (2) (1986) 196.

[2] B. Window, N. Savvides, J. Vac. Sci. Technol. A 4 (2) (1986) 453.

[3] N. Savvides, B. Window, J. Vac. Sci. Technol. A 4 (2) (1986) 504.

[4] W.D. Sproul, P.J. Rudnik, M.E. Graham, S.L. Rohde, Surf. Coat. Technol. 43-44 (1990) 270 .

[5] S.L. Rohde, I. Petrov, W.D. Sproul, S.A. Barnett, P.J. Rudnik, M.E. Graham, Thin Solid Films, 193-194 (1990) 117.

[6] R.D. Arnell, P.J. Kelly, Surf. Coat. Technol. 112 (1999) 170.

[7] U. Helmersson, M. Lattemann, J. Bohlmark, A. Ehiasarian, J. Gudmundsson, Thin Solid Films, 513 (2006) 1.

[8] D. Lundin, P. Larsson, E. Wallin, M. Lattemann, N. Brenning, U. Helmersson, Plasma Sources Sci. Technol. 17 (2008) 035021.

[9] M.A. Lieberman, A.J. Lichtenberg, Principles of Plasma Discharges and Materials Processing, John Wiley \& Sons, New York, USA, 2005, pp. 191.

[10] Software package Multiphysics 3.5 by Comsol.

[11] P. Sigurjonsson, Spatial and temporal variations of the plasma parameters in a high power impulse magnetron sputtering (HiPIMS) discharge, (Reykjavik) Faculty of Engineering, University of Iceland, 2008, pp. 17-19.

[12] F. Magnus, J.T. Gudmundsson, Rev. Sci. Instrum. 79 (2008) 073503.

[13] J. Bohlmark, A.P. Ehiasarian, P.Eh. Hovsepian, U. Helmersson, Proceeding of the 47th Annual Technical Conference of the Society of Vacuum Coaters, April 24-29, 2004, Dallas, TX, USA, pp. 432.

[14] J. Alami, P.O.Å. Persson, D. Music, J.T. Gudmundsson, J. Bohlmark, U. Helmersson, J. Vac. Sci. Technol. A 23 (2) (2005) 278.

[15] D.J. Christie, J. Vac. Sci. Technol. A 23 (2005) 330.

[16] J. Emmerlich, S. Mraz, R. Snyders, K. Jiang, J.M. Schneider, Vacuum 82 (2008) 867.

[17] S. Konstantinidis, J.P. Dauchot, M. Ganciu, M. Hecq, Appl. Phys. Lett. 88 (2006) 21501.

[18] J. Bohlmark, M. Östbye, M. Lattemann, H. Ljungcrantz, T. Rosell, U. Helmersson, Thin Solid Films 515 (2006) 1928. 
[19] S.P. Bugaev, N.N. Koval, N.S. Sochugov, A.N. Zakharov, Proceedings of the 17th International Symposium on Discharges and Electrical Insulation in Vacuum, July 21-26, 1996, Berkeley, CA, USA, pp. 1074. 


\section{Figure Captions}

Figure 1. A schematic of the dual-magnetron open field sputtering system. Both of the magnetrons are driven synchronously by HiPIMS or DC power supply. Substrates are mounted vertically on a holder placed $2 \mathrm{~cm}$ away from the edge of the magnetrons.

Figure 2. Model of the dual-magnetron system displaying an axial-symmetric cross section of the two magnetrons with the reference point marked at the far left. The contour plot shows the magnetic potential lines and the surface plot indicates the magnetic flux density.

Figure 3. Cross-sectional SEM images of the films grown at $250 \mathrm{~W}$ target power, 10 mTorr Ar gas pressure and $3 \mathrm{~cm}$ separation distance between the magnetrons with a) DCMS and b) HiPIMS.

Figure 4. Deposition rates of the film growth at $250 \mathrm{~W}$ target power and $10 \mathrm{mTorr}$ Ar gas pressure with a) DCMS and b) HiPIMS. Lines are guide for the eye only.

Figure 5. Optical emission spectra of a) DCMS and b) HiPIMS plasma discharges recorded at $250 \mathrm{~W}$ target power, $10 \mathrm{mTorr}$ Ar gas pressure and $5 \mathrm{~cm}$ separation distance between the magnetrons. The spectral lines for $\mathrm{Ti}$ and $\mathrm{Ti}+$ is predominantly found in the region 200-600 nm, whereas the Ar and Ar+ dominate the region above $600 \mathrm{~nm}$.

Figure 6. Dependence of the electron density $\left(n_{e}\right)$, on the separation distance between the magnetrons and on Ar gas pressure for a) DCMS and b) HiPIMS plasma discharges. The densities were obtained by Langmuir probe measurements at $250 \mathrm{~W}$ target power. Lines are guide for the eye only. 
Fig. 1

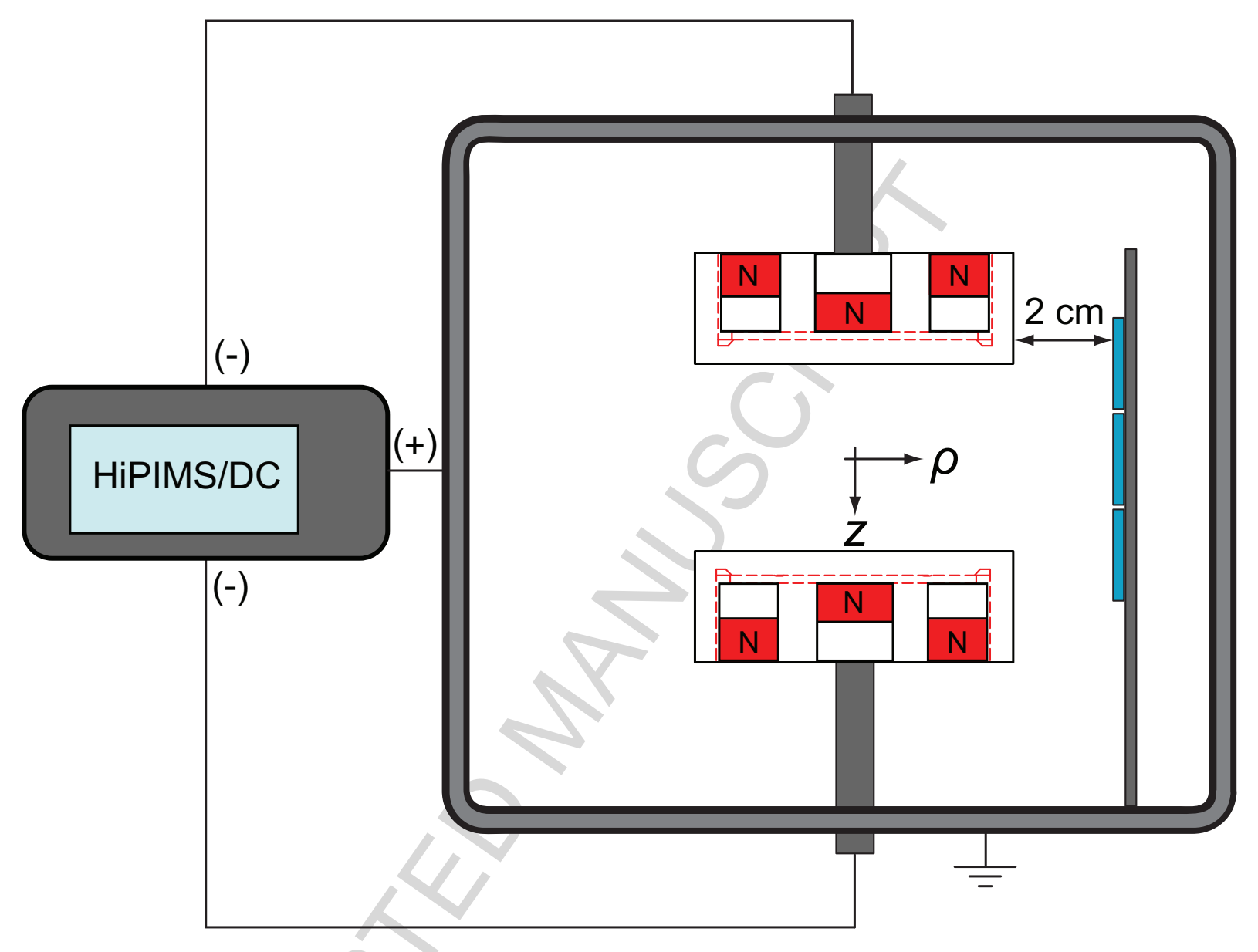

Fig. 2 


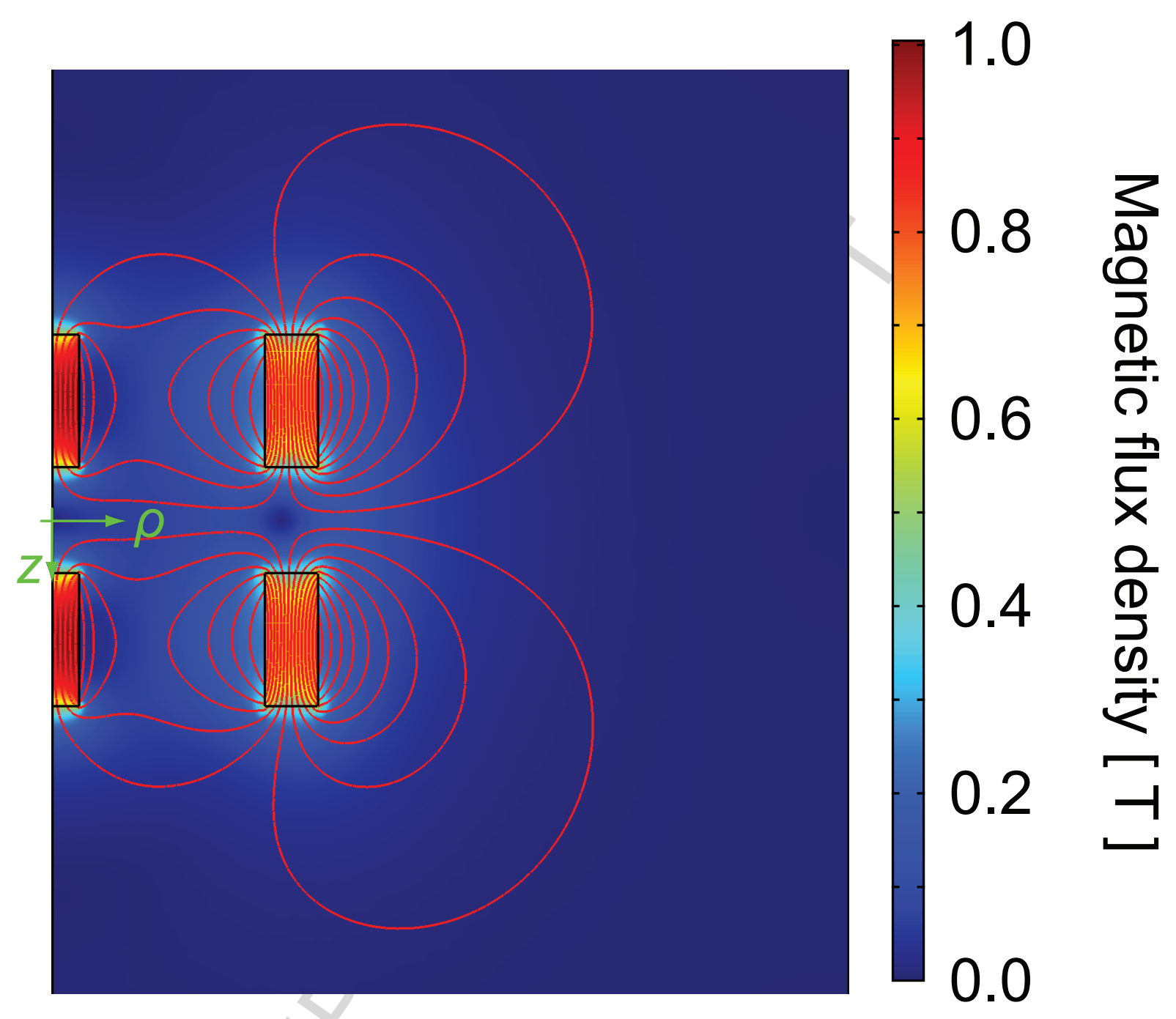

Fig. 3a

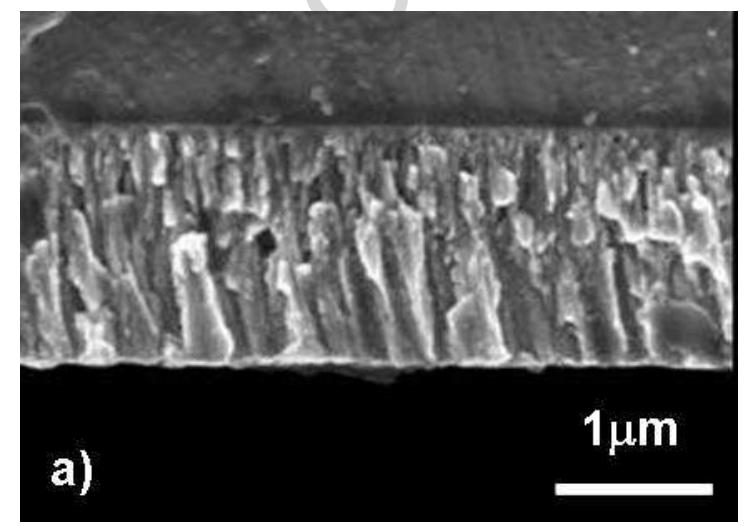

Fig. $3 b$ 


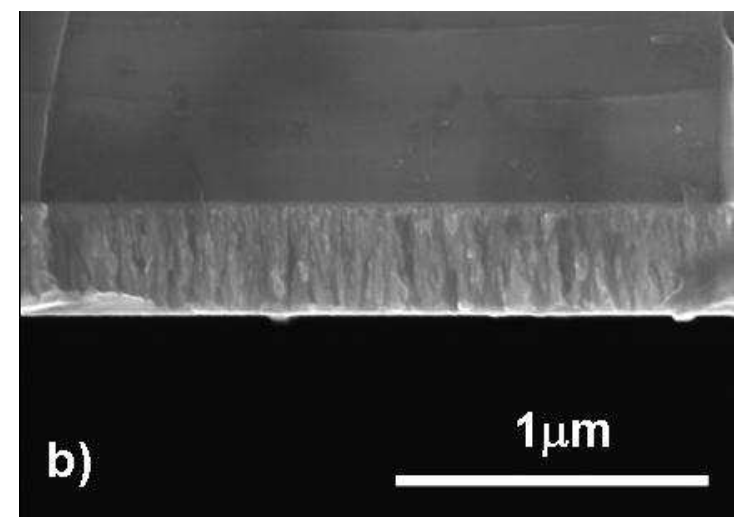

Fig. $4 \mathrm{a}$

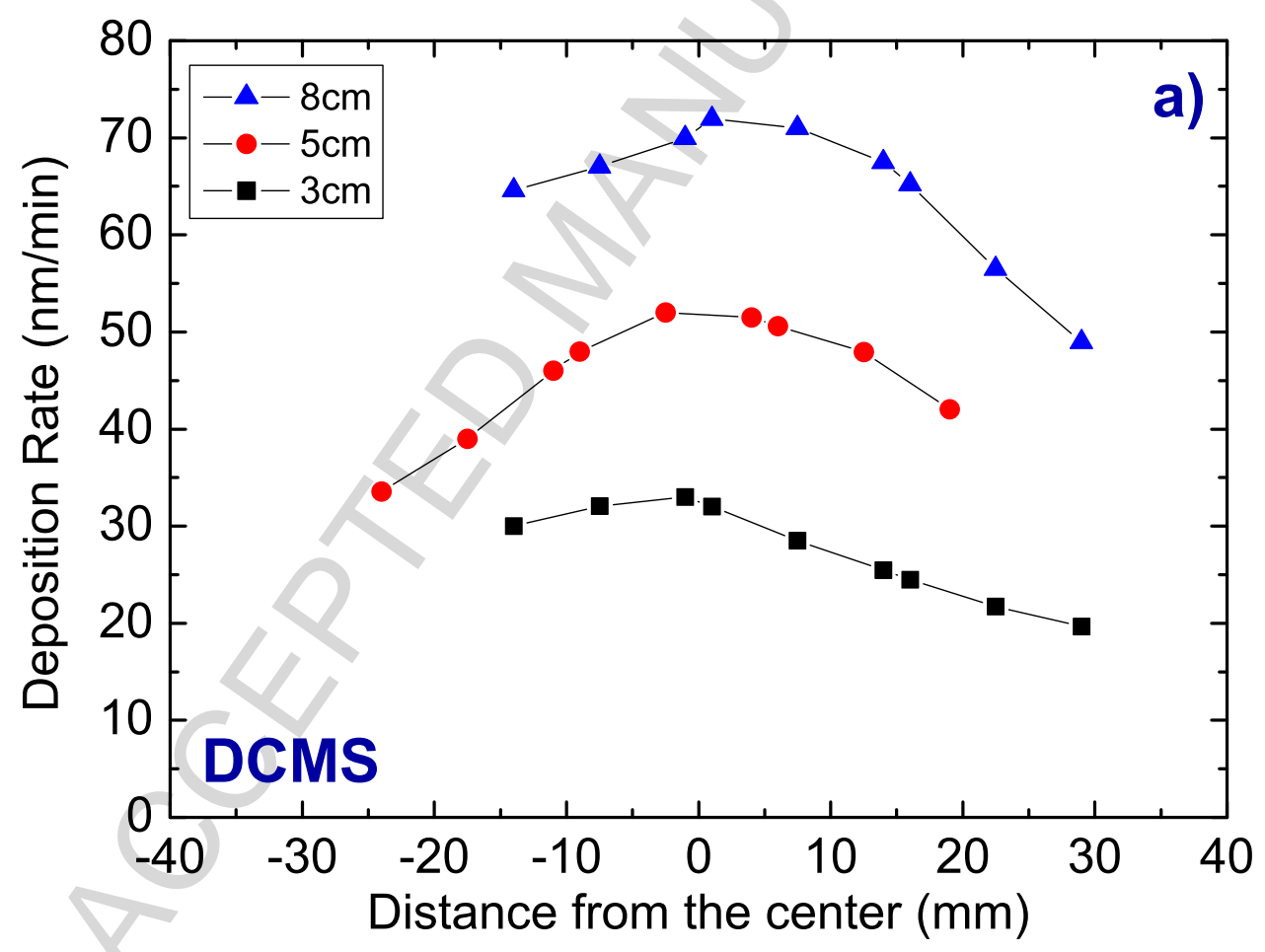

Fig. $4 b$ 


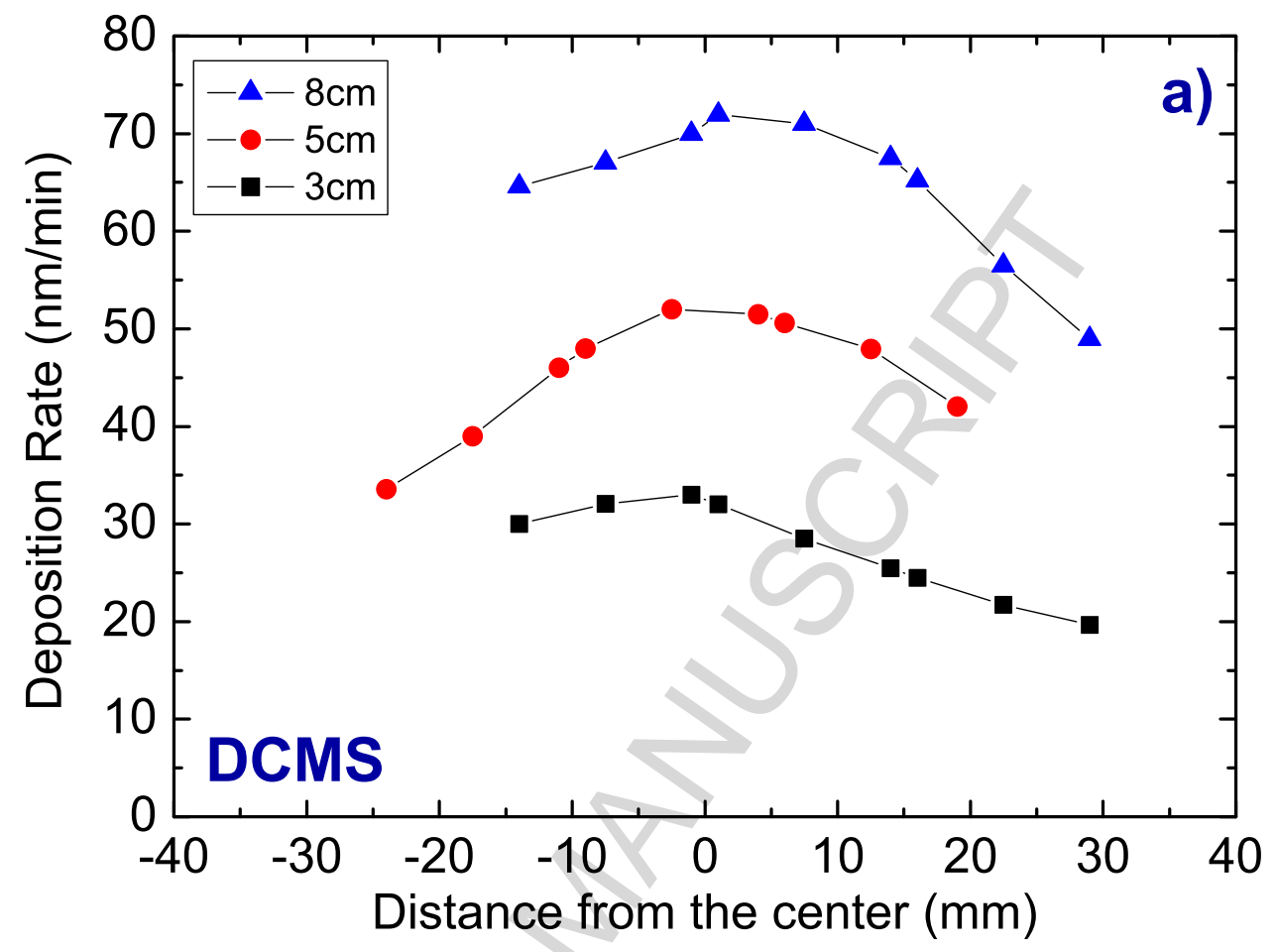

Fig. 5

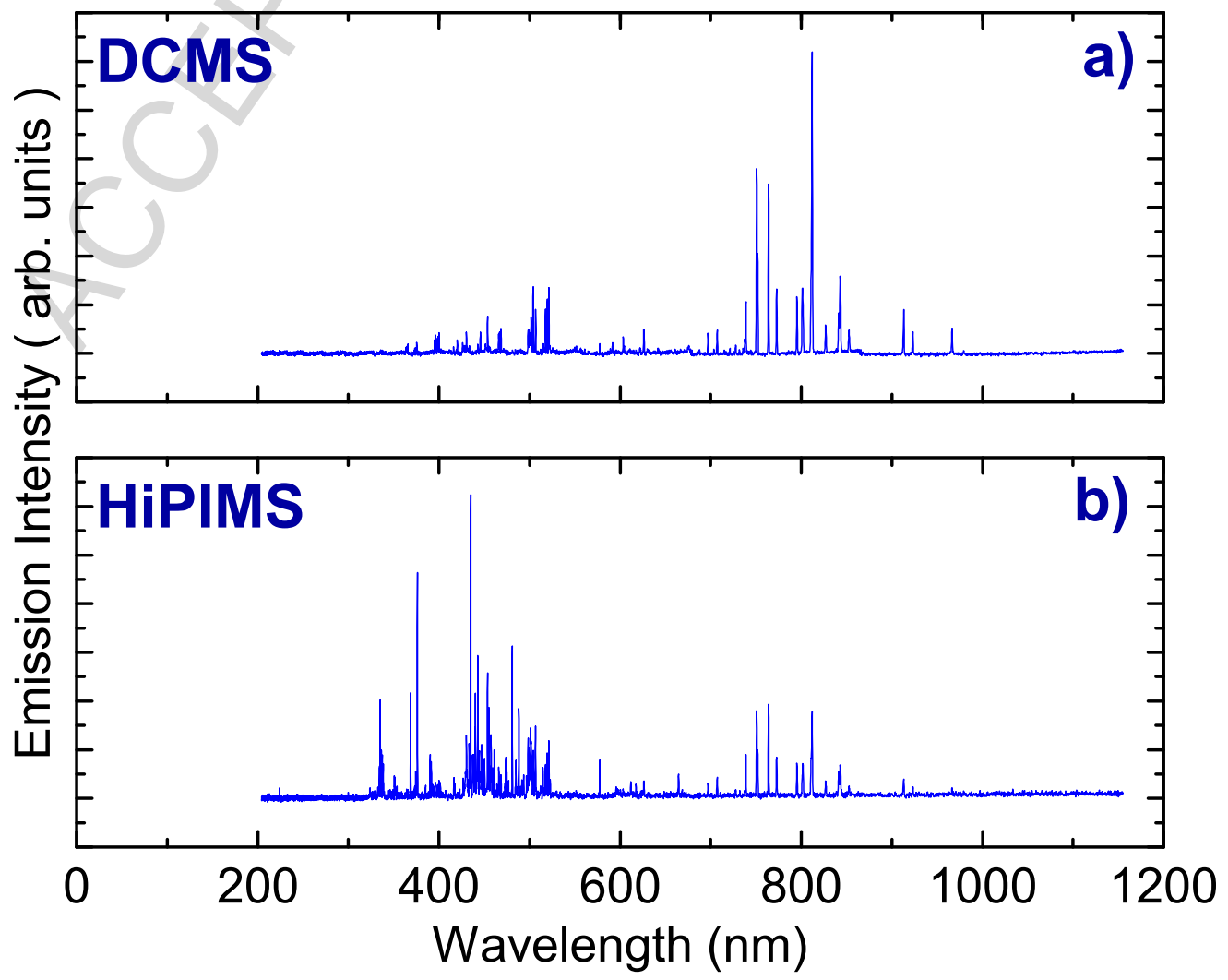


Fig. 6a

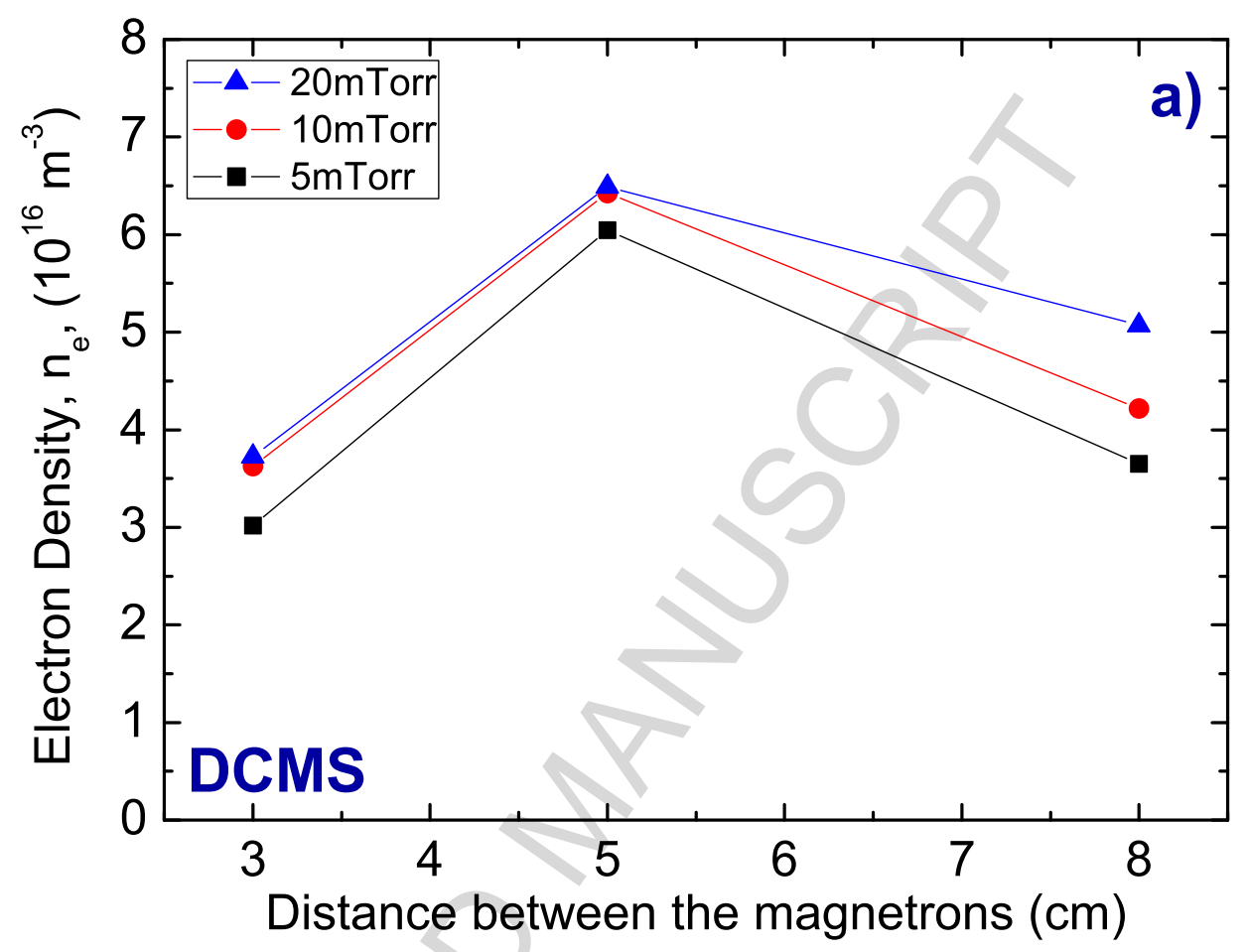

Fig. 6b

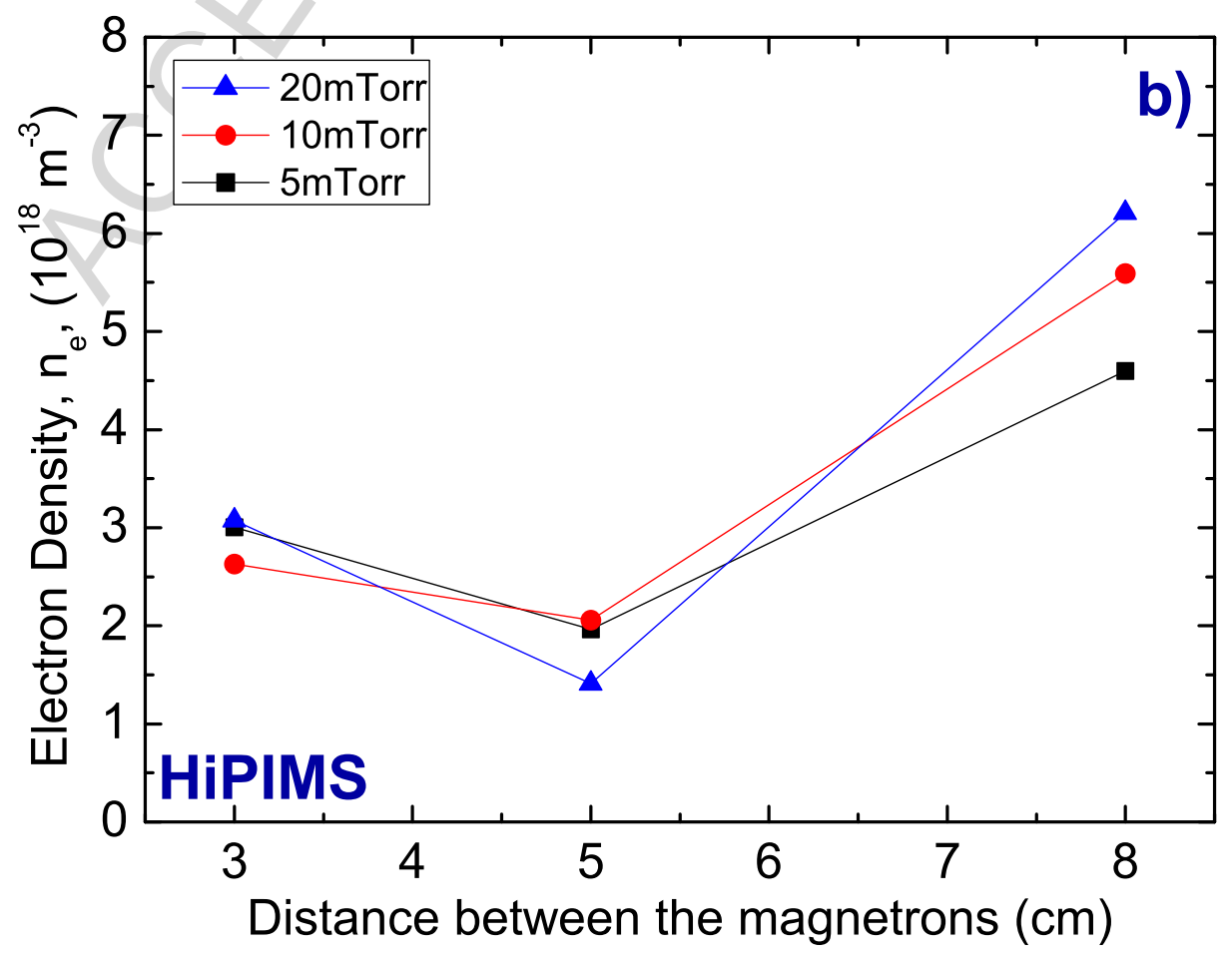

